\title{
Some new judgement theorems of Schur geometric and Schur harmonic convexities for a class of symmetric functions
}

Huan-Nan Shi ${ }^{1}$ and Jing Zhang ${ }^{2 *}$

\section{"Correspondence:}

Idtzhangjing1@buu.edu.cn

${ }^{2}$ Basic Courses Department, Beijing

Union University, Beijing, 100101,

P.R. China

Full list of author information is

available at the end of the article

\begin{abstract}
The judgement theorems of Schur geometric and Schur harmonic convexities for a class of symmetric functions are given. As their application, some analytic inequalities are established.

MSC: Primary 26D15; 05E05; 26B25

Keywords: Schur geometric convexity; Schur harmonic convexity; inequality; symmetric function
\end{abstract}

\section{Introduction}

Throughout this paper, $\mathbb{R}$ denotes the set of real numbers, $\mathbf{x}=\left(x_{1}, x_{2}, \ldots, x_{n}\right)$ denotes $n$ tuple ( $n$-dimensional real vectors), the set of vectors can be written as

$$
\begin{aligned}
& \mathbb{R}^{n}=\left\{\mathbf{x}=\left(x_{1}, \ldots, x_{n}\right): x_{i} \in \mathbb{R}, i=1, \ldots, n\right\}, \\
& \mathbb{R}_{+}^{n}=\left\{\mathbf{x}=\left(x_{1}, \ldots, x_{n}\right): x_{i}>0, i=1, \ldots, n\right\} .
\end{aligned}
$$

In particular, the notations $\mathbb{R}$ and $\mathbb{R}_{+}$denote $\mathbb{R}^{1}$ and $\mathbb{R}_{+}^{1}$, respectively.

Let $\pi=(\pi(1), \ldots, \pi(n))$ be a permutation of $(1, \ldots, n)$, all permutations are totally $n$ !. The following conclusion is proved in [1, pp.127-129].

Theorem A Let $A \subset \mathbb{R}^{k}$ be a symmetric convex set, and let $\varphi$ be a Schur-convex function defined on $A$ with the property that for each fixed $x_{2}, \ldots, x_{k}, \varphi\left(z, x_{2}, \ldots, x_{k}\right)$ is convex in $z$ on $\left\{z:\left(z, x_{2}, \ldots, x_{k}\right) \in A\right\}$. Then, for any $n>k$,

$$
\psi\left(x_{1}, \ldots, x_{n}\right)=\sum_{\pi} \varphi\left(x_{\pi(1)}, \ldots, x_{\pi(k)}\right)
$$

is Schur-convex on

$$
B=\left\{\left(x_{1}, \ldots, x_{n}\right):\left(x_{\pi(1)}, \ldots, x_{\pi(k)}\right) \in \text { A for all permutations } \pi\right\} .
$$

Furthermore, the symmetric function

$$
\bar{\psi}(\mathbf{x})=\sum_{1 \leq i_{1}<\cdots<i_{k} \leq n} \varphi\left(x_{i_{1}}, \ldots, x_{i_{k}}\right)
$$

is also Schur-convex on B.

O2013 Shi and Zhang; licensee Springer. This is an Open Access article distributed under the terms of the Creative Commons Attribution License (http://creativecommons.org/licenses/by/2.0), which permits unrestricted use, distribution, and reproduction in any medium, provided the original work is properly cited. 
Theorem A is very effective for judgement of the Schur-convexity of the symmetric functions of the form (2), see the references [1] and [2].

The Schur geometrically convex functions were proposed by Zhang [3] in 2004. Further, the Schur harmonically convex functions were proposed by Chu and Lü [4] in 2009. The theory of majorization was enriched and expanded by using these concepts [5-15]. Regarding Schur geometrically convex functions and Schur harmonically convex functions, the aim of this paper is to establish the following judgement theorems which are similar to Theorem A.

Theorem 1 Let $A \subset \mathbb{R}^{k}$ be a symmetric geometrically convex set, and let $\varphi$ be a Schur geometrically convex (concave) function defined on $A$ with the property that for each fixed $x_{2}, \ldots, x_{k}, \varphi\left(z, x_{2}, \ldots, x_{k}\right)$ is $G A$ convex (concave) in $z$ on $\left\{z:\left(z, x_{2}, \ldots, x_{k}\right) \in A\right\}$. Then, for any $n>k$,

$$
\psi\left(x_{1}, \ldots, x_{n}\right)=\sum_{\pi} \varphi\left(x_{\pi(1)}, \ldots, x_{\pi(k)}\right)
$$

is Schur geometrically convex (concave) on

$$
B=\left\{\left(x_{1}, \ldots, x_{n}\right):\left(x_{\pi(1)}, \ldots, x_{\pi(k)}\right) \in \text { A for all permutations } \pi\right\} \text {. }
$$

Furthermore, the symmetric function

$$
\bar{\psi}(\mathbf{x})=\sum_{1 \leq i_{1}<\cdots<i_{k} \leq n} \varphi\left(x_{i_{1}}, \ldots, x_{i_{k}}\right)
$$

is also Schur geometrically convex (concave) on $B$.

Theorem 2 Let $A \subset \mathbb{R}^{k}$ be a symmetric harmonically convex set, and let $\varphi$ be a Schur harmonically convex (concave) function defined on $A$ with the property that for each fixed $x_{2}, \ldots, x_{k}, \varphi\left(z, x_{2}, \ldots, x_{k}\right)$ is HA convex (concave) in $z$ on $\left\{z:\left(z, x_{2}, \ldots, x_{k}\right) \in A\right\}$. Then, for any $n>k$,

$$
\psi\left(x_{1}, \ldots, x_{n}\right)=\sum_{\pi} \varphi\left(x_{\pi(1)}, \ldots, x_{\pi(k)}\right)
$$

is Schur harmonically convex (concave) on

$$
B=\left\{\left(x_{1}, \ldots, x_{n}\right):\left(x_{\pi(1)}, \ldots, x_{\pi(k)}\right) \in \text { A for all permutations } \pi\right\} \text {. }
$$

Furthermore, the symmetric function

$$
\bar{\psi}(\mathbf{x})=\sum_{1 \leq i_{1}<\cdots<i_{k} \leq n} \varphi\left(x_{i_{1}}, \ldots, x_{i_{k}}\right)
$$

is also Schur harmonically convex (concave) on B. 


\section{Definitions and lemmas}

In order to prove some further results, in this section we recall useful definitions and lemmas.

Definition $1[1,16]$ Let $\mathbf{x}=\left(x_{1}, \ldots, x_{n}\right)$ and $\mathbf{y}=\left(y_{1}, \ldots, y_{n}\right) \in \mathbb{R}^{n}$.

(i) We say $\mathbf{y}$ majorizes $\mathbf{x}$ ( $\mathbf{x}$ is said to be majorized by $\mathbf{y}$ ), denoted by $\mathbf{x} \prec \mathbf{y}$, if $\sum_{i=1}^{k} x_{[i]} \leq \sum_{i=1}^{k} y_{[i]}$ for $k=1,2, \ldots, n-1$ and $\sum_{i=1}^{n} x_{i}=\sum_{i=1}^{n} y_{i}$, where $x_{[1]} \geq \cdots \geq x_{[n]}$ and $y_{[1]} \geq \cdots \geq y_{[n]}$ are rearrangements of $\mathbf{x}$ and $\mathbf{y}$ in a descending order.

(ii) Let $\Omega \subset \mathbb{R}^{n}$, a function $\varphi: \Omega \rightarrow \mathbb{R}$ is said to be a Schur-convex function on $\Omega$ if $\mathbf{x} \prec \mathbf{y}$ on $\Omega$ implies $\varphi(\mathbf{x}) \leq \varphi(\mathbf{y})$. A function $\varphi$ is said to be a Schur-concave function on $\Omega$ if and only if $-\varphi$ is Schur-convex function on $\Omega$.

Definition $2[1,16]$ Let $\mathbf{x}=\left(x_{1}, \ldots, x_{n}\right)$ and $\mathbf{y}=\left(y_{1}, \ldots, y_{n}\right) \in \mathbb{R}^{n}, 0 \leq \alpha \leq 1$. A set $\Omega \subset \mathbb{R}^{n}$ is said to be a convex set if $\mathbf{x}, \mathbf{y} \in \Omega$ implies $\alpha \mathbf{x}+(1-\alpha) \mathbf{y}=\left(\alpha x_{1}+(1-\alpha) y_{1}, \ldots, \alpha x_{n}+(1-\alpha) y_{n}\right) \in$ $\Omega$.

Definition $3[1,16]$

(i) A set $\Omega \subset \mathbb{R}^{n}$ is called a symmetric set if $\mathbf{x} \in \Omega$ implies $\mathbf{x} P \in \Omega$ for every $n \times n$ permutation matrix $P$.

(ii) A function $\varphi: \Omega \rightarrow \mathbb{R}$ is called symmetric if for every permutation matrix $P$, $\varphi(\mathbf{x} P)=\varphi(\mathbf{x})$ for all $\mathbf{x} \in \Omega$.

Definition 4 Let $\Omega \subset \mathbb{R}_{+}^{n}, \mathbf{x}=\left(x_{1}, \ldots, x_{n}\right) \in \Omega$ and $\mathbf{y}=\left(y_{1}, \ldots, y_{n}\right) \in \Omega$.

(i) [3, p.64] A set $\Omega$ is called a geometrically convex set if $\left(x_{1}^{\alpha} y_{1}^{\beta}, \ldots, x_{n}^{\alpha} y_{n}^{\beta}\right) \in \Omega$ for all $\mathbf{x}, \mathbf{y} \in \Omega$ and $\alpha, \beta \in[0,1]$ such that $\alpha+\beta=1$.

(ii) [3, p.107] A function $\varphi: \Omega \rightarrow \mathbb{R}_{+}$is said to be a Schur geometrically convex function on $\Omega$ if $\left(\log x_{1}, \ldots, \log x_{n}\right) \prec\left(\log y_{1}, \ldots, \log y_{n}\right)$ on $\Omega$ implies $\varphi(\mathbf{x}) \leq \varphi(\mathbf{y})$. A function $\varphi$ is said to be a Schur geometrically concave function on $\Omega$ if and only if $-\varphi$ is a Schur geometrically convex function.

Definition 5 [17] Let $\Omega \subset \mathbb{R}_{+}^{n}$.

(i) A set $\Omega$ is said to be a harmonically convex set if $\frac{\mathbf{x y}}{\lambda \mathbf{x}+(1-\lambda) \mathbf{y}} \in \Omega$ for every $\mathbf{x}, \mathbf{y} \in \Omega$ and $\lambda \in[0,1]$, where $\mathbf{x y}=\sum_{i=1}^{n} x_{i} y_{i}$ and $\frac{1}{\mathbf{x}}=\left(\frac{1}{x_{1}}, \ldots, \frac{1}{x_{n}}\right)$.

(ii) A function $\varphi: \Omega \rightarrow \mathbb{R}_{+}$is said to be a Schur harmonically convex function on $\Omega$ if $\frac{1}{\mathbf{x}} \prec \frac{1}{\mathbf{y}}$ implies $\varphi(\mathbf{x}) \leq \varphi(\mathbf{y})$. A function $\varphi$ is said to be a Schur harmonically concave function on $\Omega$ if and only if $-\varphi$ is a Schur harmonically convex function.

Definition 6 [18] Let $I \subset \mathbb{R}_{+}, \varphi: I \rightarrow \mathbb{R}_{+}$be continuous.

(i) A function $\varphi$ is said to be a GA convex (concave) function on $I$ if

$$
\varphi(\sqrt{x y}) \leq(\geq) \frac{\varphi(x)+\varphi(y)}{2}
$$

for all $x, y \in I$.

(ii) A function $\varphi$ is said to be a HA convex (concave) function on $I$ if

$$
\varphi\left(\frac{2 x y}{x+y}\right) \leq(\geq) \frac{\varphi(x)+\varphi(y)}{2}
$$

for all $x, y \in I$. 
Lemma 1 [16, p.57] Let $\Omega \subset \mathbb{R}^{n}$ be a symmetric convex set with a nonempty interior $\Omega^{0}$. $\varphi: \Omega \rightarrow \mathbb{R}$ is continuous on $\Omega$ and differentiable on $\Omega^{0}$. Then $\varphi$ is a Schur-convex (Schurconcave) function if and only if $\varphi$ is symmetric on $\Omega$ and

$$
\left(x_{1}-x_{2}\right)\left(\frac{\partial \varphi}{\partial x_{1}}-\frac{\partial \varphi}{\partial x_{2}}\right) \geq 0(\leq 0)
$$

holds for any $\mathbf{x}=\left(x_{1}, \ldots, x_{n}\right) \in \Omega^{0}$.

Lemma 2 [3, p.108] Let $\Omega \subset \mathbb{R}_{+}^{n}$ be a symmetric geometrically convex set with a nonempty interior $\Omega^{0}$. Let $\varphi: \Omega \rightarrow \mathbb{R}_{+}$be continuous on $\Omega$ and differentiable on $\Omega^{0}$. Then $\varphi$ is a Schur geometrically convex (Schur geometrically concave) function if and only if $\varphi$ is symmetric on $\Omega$ and

$$
\left(x_{1}-x_{2}\right)\left(x_{1} \frac{\partial \varphi}{\partial x_{1}}-x_{2} \frac{\partial \varphi}{\partial x_{2}}\right) \geq 0(\leq 0)
$$

holds for any $\mathbf{x}=\left(x_{1}, \ldots, x_{n}\right) \in \Omega^{0}$.

Lemma $3[17,19]$ Let $\Omega \subset \mathbb{R}_{+}^{n}$ be a symmetric harmonically convex set with a nonempty interior $\Omega^{0}$. Let $\varphi: \Omega \rightarrow \mathbb{R}_{+}$be continuous on $\Omega$ and differentiable on $\Omega^{0}$. Then $\varphi$ is a Schur harmonically convex (Schur harmonically concave) function if and only if $\varphi$ is symmetric on $\Omega$ and

$$
\left(x_{1}-x_{2}\right)\left(x_{1}^{2} \frac{\partial \varphi}{\partial x_{1}}-x_{2}^{2} \frac{\partial \varphi}{\partial x_{2}}\right) \geq 0(\leq 0)
$$

holds for any $\mathbf{x}=\left(x_{1}, \ldots, x_{n}\right) \in \Omega^{0}$.

Lemma 4 [18] Let $I \subset \mathbb{R}_{+}$be an open subinterval, and let $\varphi: I \rightarrow \mathbb{R}_{+}$be differentiable.

(i) $\varphi$ is GA-convex (concave) if and only if $x \varphi^{\prime}(x)$ is increasing (decreasing).

(ii) $\varphi$ is HA-convex (concave) if and only if $x^{2} \varphi^{\prime}(x)$ is increasing (decreasing).

\section{Proofs of main results}

Proof of Theorem 1 To verify condition (4) of Lemma 2, denote by $\sum_{\pi(i, j)}$ the summation over all permutations $\pi$ such that $\pi(i)=1, \pi(j)=2$. Because $\varphi$ is symmetric,

$$
\begin{aligned}
\psi\left(x_{1}, \ldots, x_{n}\right) \\
=\sum_{\substack{i, j \leq k \\
i \neq j}} \sum_{\pi(i, j)} \varphi\left(x_{1}, x_{2}, x_{\pi(1)}, \ldots, x_{\pi(i-1)}, x_{\pi(i+1)}, \ldots, x_{\pi(j-1)}, x_{\pi(j+1)}, \ldots, x_{\pi(k)}\right) \\
\quad+\sum_{i \leq k<j} \sum_{\pi(i, j)} \varphi\left(x_{1}, x_{\pi(1)}, \ldots, x_{\pi(i-1)}, x_{\pi(i+1)}, \ldots, x_{\pi(k)}\right) \\
\quad+\sum_{j \leq k<i \pi(i, j)} \varphi\left(x_{2}, x_{\pi(1)}, \ldots, x_{\pi(j-1)}, x_{\pi(j+1)}, \ldots, x_{\pi(k)}\right) \\
\quad+\sum_{k<i, j} \sum_{\pi(i, j)} \varphi\left(x_{\pi(1)}, \ldots, x_{\pi(k)}\right) .
\end{aligned}
$$


Then

$$
\begin{aligned}
\Delta_{1}:= & \left(x_{1} \frac{\partial \psi}{\partial x_{1}}-x_{2} \frac{\partial \psi}{\partial x_{2}}\right)\left(x_{1}-x_{2}\right) \\
= & \sum_{\substack{i, j \leq k \\
i \neq j}} \sum_{\pi(i, j)}\left[x_{1} \varphi_{(1)}\left(x_{1}, x_{2}, x_{\pi(1)}, \ldots, x_{\pi(i-1)}, x_{\pi(i+1)}, \ldots, x_{\pi(j-1)}, x_{\pi(j+1)}, \ldots, x_{\pi(k)}\right)\right. \\
& \left.-x_{2} \varphi_{(2)}\left(x_{1}, x_{2}, x_{\pi(1)}, \ldots, x_{\pi(i-1)}, x_{\pi(i+1)}, \ldots, x_{\pi(j-1)}, x_{\pi(j+1)}, \ldots, x_{\pi(k)}\right)\right]\left(x_{1}-x_{2}\right) \\
& +\sum_{i \leq k<j \pi(i, j)} \sum_{1}\left[x_{1} \varphi_{(1)}\left(x_{1}, x_{\pi(1)}, \ldots, x_{\pi(i-1)}, x_{\pi(i+1)}, \ldots, x_{\pi(k)}\right)\right. \\
& \left.-x_{2} \varphi_{(1)}\left(x_{2}, x_{\pi(1)}, \ldots, x_{\pi(i-1)}, x_{\pi(i+1)}, \ldots, x_{\pi(k)}\right)\right]\left(x_{1}-x_{2}\right) .
\end{aligned}
$$

Here,

$$
\left(x_{1} \varphi_{(1)}-x_{2} \varphi_{(2)}\right)\left(x_{1}-x_{2}\right) \geq 0(\leq 0)
$$

because $\varphi$ is Schur geometrically convex (concave), and

$$
\left[x_{1} \varphi_{(1)}\left(x_{1}, z\right)-x_{2} \varphi_{(1)}\left(x_{2}, z\right)\right]\left(x_{1}-x_{2}\right) \geq 0(\leq 0)
$$

because $\varphi\left(z, x_{2}, \ldots, x_{k}\right)$ is GA convex (concave) in its first argument on $\left\{z:\left(z, x_{2}, \ldots, x_{k}\right) \in\right.$ $A$ \}. Accordingly, $\Delta_{1} \geq 0(\leq 0)$. This shows that $\psi$ is Schur geometrically convex (concave) on

$$
B=\left\{\left(x_{1}, \ldots, x_{n}\right):\left(x_{\pi(1)}, \ldots, x_{\pi(k)}\right) \in \text { A for all permutations } \pi\right\} .
$$

Notice that

$$
\bar{\psi}(\mathbf{x})=\psi(\mathbf{x}) / k !(n-k) !
$$

Of course, $\bar{\psi}$ is Schur geometrically convex (concave) whenever $\psi$ is Schur geometrically convex (concave).

The proof of Theorem 1 is completed.

Proof of Theorem 2 We only need to verify condition (5) of Lemma 3, the proof is similar to that of Theorem 1 and is omitted.

Remark 1 In most applications, $A$ has the form $I^{k}$ for some interval $I \subset R$ and in this case $B=I^{n}$. Notice that the convexity of $\varphi$ in its first argument also implies that $\varphi$ is convex in each argument, the other arguments being fixed, because $\varphi$ is symmetric.

\section{Applications}

Let

$$
E_{k}\left(\frac{\mathbf{x}}{1-\mathbf{x}}\right)=\sum_{1 \leq i_{1}<\cdots<i_{k} \leq n} \prod_{j=1}^{k} \frac{x_{i_{j}}}{1-x_{i_{j}}} .
$$

In 2011, Guan and Guan [20] proved the following theorem through Lemma 2. 
Theorem 3 The symmetric function $E_{k}\left(\frac{\mathbf{x}}{1-\mathbf{x}}\right), k=1, \ldots, n$, is Schur geometrically convex on $(0,1)^{n}$.

Now, we give a new proof of Theorem 3 by using Theorem 1. Furthermore, we prove the following theorem through Theorem 2 .

Theorem 4 The symmetric function $E_{k}\left(\frac{\mathbf{x}}{1-\mathbf{x}}\right), k=1, \ldots, n$, is Schur harmonically convex on $(0,1)^{n}$.

Proof of Theorem 3 Let $\varphi(\mathbf{z})=\prod_{i=1}^{k}\left[z_{i} /\left(1-z_{i}\right)\right]$. Then

$$
\log \varphi(\mathbf{z})=\sum_{i=1}^{k}\left[\log z_{i}-\log \left(1-z_{i}\right)\right]
$$

and

$$
\begin{aligned}
\frac{\partial \varphi(\mathbf{z})}{\partial z_{1}}=\varphi(\mathbf{z})\left(\frac{1}{z_{1}}+\frac{1}{1-z_{1}}\right), \quad \frac{\partial \varphi(\mathbf{z})}{\partial z_{2}}=\varphi(\mathbf{z})\left(\frac{1}{z_{2}}+\frac{1}{1-z_{2}}\right) \\
\Delta:=\left(z_{1}-z_{2}\right)\left(z_{1} \frac{\partial \varphi(\mathbf{z})}{\partial z_{1}}-z_{2} \frac{\partial \varphi(\mathbf{z})}{\partial z_{2}}\right) \\
\quad=\left(z_{1}-z_{2}\right) \varphi(\mathbf{z})\left(\frac{z_{1}}{1-z_{1}}-\frac{z_{2}}{1-z_{2}}\right) \\
=\left(z_{1}-z_{2}\right)^{2} \varphi(\mathbf{z}) \frac{1}{\left(1-z_{2}\right)\left(1-z_{1}\right)} .
\end{aligned}
$$

This shows that $\Delta \geq 0$ when $0<z_{i}<1, i=1, \ldots, k$. According to Lemma 2, $\varphi$ is Schur geometrically convex on $A=\left\{\mathbf{z}: \mathbf{z} \in(0,1)^{k}\right\}$. Let $g(t)=\frac{t}{1-t}$, then $h(t):=\operatorname{tg}^{\prime}(t)=\frac{t}{(1-t)^{2}}$. From $t \in(0,1)$, it follows that $h^{\prime}(t)=\frac{1+t}{(1-t)^{3}} \geq 0$. According to Lemma 4(i), $\varphi$ is GA convex in its single variable on $(0,1)$. So $E_{k}\left(\frac{\mathbf{x}}{1-\mathbf{x}}\right)$ is Schur geometrically convex on $(0,1)^{n}$ from Theorem 1. The proof of Theorem 3 is completed.

Proof of Theorem 4 Let $\varphi(\mathbf{z})=\prod_{i=1}^{k}\left(z_{i} / 1-z_{i}\right)$, then

$$
\log \varphi(\mathbf{z})=\sum_{i=1}^{k}\left[\log z_{i}-\log \left(1-z_{i}\right)\right]
$$

From (7), we get

$$
\begin{aligned}
\Delta_{1} & :=\left(z_{1}-z_{2}\right)\left(z_{1}^{2} \frac{\partial \varphi(\mathbf{z})}{\partial z_{1}}-z_{2}^{2} \frac{\partial \varphi(\mathbf{z})}{\partial z_{2}}\right) \\
& =\left(z_{1}-z_{2}\right) \varphi(\mathbf{z})\left(z_{1}-z_{2}+\frac{z_{1}^{2}}{1-z_{1}}-\frac{z_{2}^{2}}{1-z_{2}}\right) \\
& =\left(z_{1}-z_{2}\right)^{2} \varphi(\mathbf{z})\left[1+\frac{z_{1}+z_{2}-z_{1} z_{2}}{\left(1-z_{2}\right)\left(1-z_{1}\right)}\right] .
\end{aligned}
$$

This shows that $\Delta_{1} \geq 0$ when $0<z_{i}<1, i=1, \ldots, k$. According to Lemma $3, \varphi$ is Schur harmonically convex on $A=\left\{\mathbf{z}: \mathbf{z} \in(0,1)^{k}\right\}$. Let $g(t)=\frac{t}{1-t}$, then $p(t):=t^{2} g^{\prime}(t)=\frac{t^{2}}{(1-t)^{2}}$. From 
$t \in(0,1)$, it follows that $p^{\prime}(t)=\frac{2 t}{(1-t)^{3}} \geq 0$. According to Lemma 4(ii), $\varphi$ is HA convex in its single variable on $(0,1)$. So $E_{k}\left(\frac{\mathbf{x}}{1-\mathbf{x}}\right)$ is Schur harmonically convex on $(0,1)^{n}$ from Theorem 2. The proof of Theorem 4 is completed.

By using Theorem A, the following conclusion is proved in [1, p.129].

The symmetric function

$$
\bar{\psi}(\mathbf{x})=\sum_{1 \leq i_{1}<\cdots<i_{k} \leq n} \frac{x_{i_{1}}+\cdots+x_{i_{k}}}{x_{i_{1}} \cdots x_{i_{k}}}
$$

is Schur-convex on $\mathbb{R}_{+}^{n}$.

Now we use Theorem 1 and Theorem 2, respectively, to study Schur geometric convexity and Schur harmonic convexity of $\bar{\psi}(\mathbf{x})$.

Theorem 5 The symmetric function $\bar{\psi}(\mathbf{x})$ is Schur geometrically convex and Schur harmonically concave on $\mathbb{R}_{+}^{n}$.

Proof Let $\varphi(\mathbf{y})=\sum_{i=1}^{k} y_{i} / \prod_{i=1}^{k} y_{i}$, then $\log \varphi(\mathbf{y})=\log \left(\sum_{i=1}^{k} y_{i}\right)-\sum_{i=1}^{k} \log y_{i}$. Thus,

$$
\begin{aligned}
& \frac{\partial \varphi(\mathbf{y})}{\partial y_{1}}=\varphi(\mathbf{y})\left(\frac{1}{\sum_{i=1}^{k} y_{i}}-\frac{1}{y_{1}}\right), \quad \frac{\partial \varphi(\mathbf{y})}{\partial y_{2}}=\varphi(\mathbf{y})\left(\frac{1}{\sum_{i=1}^{k} y_{i}}-\frac{1}{y_{2}}\right) \\
& \Delta:=\left(y_{1}-y_{2}\right)\left(y_{1} \frac{\partial \varphi(\mathbf{y})}{\partial y_{1}}-y_{2} \frac{\partial \varphi(\mathbf{y})}{\partial y_{2}}\right) \\
& =\left(y_{1}-y_{2}\right) \varphi(\mathbf{y})\left(\frac{y_{1}-y_{2}}{\sum_{i=1}^{k} y_{i}}\right) \\
& =\frac{\left(y_{1}-y_{2}\right)^{2}}{\prod_{i=1}^{k} y_{i}} \geq 0 .
\end{aligned}
$$

According to Lemma 2, $\varphi(\mathbf{y})$ is Schur geometrically convex on $\mathbb{R}_{+}^{k}$. Let $g(z)=\varphi\left(z, x_{2}, \ldots\right.$, $\left.x_{k}\right)=\frac{z+a}{b z}=\frac{1}{b}+\frac{a}{b z}$, where $a=\sum_{i=2}^{k} x_{i}, b=\prod_{i=2}^{k} x_{i}$, then $h(z):=z g^{\prime}(z)=-\frac{a}{b z}$. From $z \in \mathbb{R}_{+}$, it follows that $h^{\prime}(z)=\frac{a}{b z^{2}} \geq 0$. According to Lemma 4(i), $\varphi$ is GA convex in its single variable on $\mathbb{R}_{+}$. So $\bar{\psi}(\mathbf{x})$ is Schur geometrically convex on $\mathbb{R}_{+}$from Theorem 1 .

It is easy to check that

$$
\begin{aligned}
\Delta_{1} & :=\left(y_{1}-y_{2}\right)\left(y_{1}^{2} \frac{\partial \varphi(\mathbf{y})}{\partial y_{1}}-y_{2}^{2} \frac{\partial \varphi(\mathbf{y})}{\partial y_{2}}\right) \\
& =\frac{\left(y_{1}-y_{2}\right)^{2}\left(y_{1}+y_{2}-\sum_{i=1}^{k} y_{i}\right)}{\prod_{i=1}^{k} y_{i}} \leq 0 .
\end{aligned}
$$

According to Lemma 3, $\varphi(\mathbf{y})$ is Schur harmonically concave on $\mathbb{R}_{+}^{k}$. Let $h(z):=z^{2} g^{\prime}(z)=-\frac{a}{b}$. $h^{\prime}(z)=0$ when $z \in \mathbb{R}_{+}$. According to Lemma 4(ii), $\varphi$ is HA concave in its single variable on $\mathbb{R}_{+}$. So $\bar{\psi}(\mathbf{x})$ is Schur harmonically concave on $\mathbb{R}_{+}^{n}$ from Theorem 2 .

Remark 2 Let

$$
H=\frac{n}{\sum_{i=1}^{n} \frac{1}{x_{i}}}, \quad G=\left(\prod_{i=1}^{n} x_{i}\right)^{\frac{1}{n}}
$$


where $x_{i}>0, i=1, \ldots, n$. Then

$$
\begin{aligned}
& (\log G, \ldots, \log G) \prec\left(\log x_{1}, \ldots, \log x_{n}\right), \\
& \left(\frac{1}{H}, \ldots, \frac{1}{H}\right) \prec\left(\frac{1}{x_{1}}, \ldots, \frac{1}{x_{n}}\right) .
\end{aligned}
$$

From Theorem 5, it follows that

$$
\frac{k C_{n}^{k}}{H^{k-1}} \geq \sum_{1 \leq i_{1}<\cdots<i_{k} \leq n} \frac{x_{i_{1}}+\cdots+x_{i_{k}}}{x_{i_{1}} \cdots x_{i_{k}}} \geq \frac{k C_{n}^{k}}{G^{k-1}} .
$$

By using Theorem A, the following conclusion is proved in [1, p.129].

The symmetric function

$$
\psi(\mathbf{x})=\sum_{1 \leq i_{1}<\cdots<i_{k} \leq n} \frac{x_{i_{1}} \cdots x_{i_{k}}}{x_{i_{1}}+\cdots+x_{i_{k}}}
$$

is Schur-concave on $\mathbb{R}_{+}^{n}$.

By applying Theorem 2, we further obtain the following result.

Theorem 6 The symmetric function $\psi(\mathbf{x})$ is Schur harmonically convex on $\mathbb{R}_{+}^{n}$.

Proof Let $\lambda(\mathbf{y})=\prod_{i=1}^{k} y_{i} / \sum_{i=1}^{k} y_{i}$. According to the proof of Theorem 5, $\varphi(\mathbf{y})$ is Schur harmonically concave on $\mathbb{R}_{+}^{k}$. Let $\lambda(\mathbf{y})=\frac{1}{\varphi(\mathbf{y})}$. From the definition of Schur harmonically convex, it follows that $\lambda(\mathbf{y})$ is Schur harmonically convex on $\mathbb{R}_{+}^{k}$. Let $g(z)=\lambda\left(z, x_{2}, \ldots, x_{k}\right)=\frac{b z}{z+a}$, where $a=\sum_{i=2}^{k} x_{i}, b=\prod_{i=2}^{k} x_{i}$. Then $h(z):=z^{2} g^{\prime}(z)=\frac{z^{2} a b}{(z+a)^{2}}$. With the fact that $h^{\prime}(z)=$ $\frac{2 z a^{2} b}{(z+a)^{3}} \geq 0$ for $z \in \mathbb{R}_{+}$, it follows that $\varphi$ is HA convex in its single variable on $\mathbb{R}_{+}$. So, from Theorem $2, \psi(\mathbf{x})$ is Schur harmonically convex on $\mathbb{R}_{+}^{n}$.

Remark 3 From Theorem 6 and (10), it follows that

$$
\sum_{1 \leq i_{1}<\cdots<i_{k} \leq n} \frac{x_{i_{1}} \cdots x_{i_{k}}}{x_{i_{1}}+\cdots+x_{i_{k}}} \geq \frac{H^{k-1} C_{n}^{k}}{k}
$$

where $x_{i}>0, i=1, \ldots, n$.

Remark 4 It needs further discussion that $\psi(\mathbf{x})$ is Schur geometrically convex on $\mathbb{R}_{+}^{n}$.

\section{Competing interests}

The authors declare that they have no competing interests.

\section{Authors' contributions}

The authors co-authored this paper together. All authors read and approved the final manuscript.

\section{Author details}

${ }^{1}$ Department of Electronic Information, Teacher's College, Beijing Union University, Beijing, 100011, P.R. China. ${ }^{2}$ Basic

Courses Department, Beijing Union University, Beijing, 100101, P.R. China. 


\section{Acknowledgements}

The work was supported by the Funding Project for Academic Human Resources Development in Institutions of Higher Learning under the Jurisdiction of Beijing Municipality (PHR (IHLB)) (PHR201108407) and the National Natural Science Foundation of China (Grant No. 11101034).

Received: 22 July 2013 Accepted: 18 October 2013 Published: 11 Nov 2013

\section{References}

1. Marshall, AW, Olkin, I, Arnold, BC: Inequalities: Theory of Majorization and Its Application, 2nd edn. Springer, New York (2011)

2. Shi, H-N: Schur convexity of three symmetric functions. J. Hexi Univ. 27(2), 13-17 (2011) (in Chinese)

3. Zhang, XM: Geometrically Convex Functions. An'hui University Press, Hefei (2004) (in Chinese)

4. Chu, Y-M, Lü, Y-P: The Schur harmonic convexity of the Hamy symmetric function and its applications. J. Inequal. Appl. 2009, Article ID 838529 (2009). doi:10.1155/2009/838529

5. Xia, W-F, Chu, Y-M: Schur-convexity for a class of symmetric functions and its applications. J. Inequal. Appl. 2009, Article ID 493759 (2009). doi:10.1155/2009/493759

6. Rovența, I: Schur convexity of a class of symmetric functions. An. Univ. Craiova, Ser. Mat. Inform. 37(1), 12-18 (2010)

7. Xia, W-F, Chu, Y-M: On Schur convexity of some symmetric functions. J. Inequal. Appl. 2010, Article ID 543250 (2010). doi:10.1155/2010/543250

8. Meng, J, Chu, Y, Tang, X: The Schur-harmonic-convexity of dual form of the Hamy symmetric function. Mat. Vesn. 62(1), 37-46 (2010)

9. Chu, Y-M, Wang, G-D, Zhang, X-H: The Schur multiplicative and harmonic convexities of the complete symmetric function. Math. Nachr. 284(5-6), 653-663 (2011)

10. Chu, Y-M, Xia, W-F, Zhao, T-H: Some properties for a class of symmetric functions and applications. J. Math. Inequal. 5(1), 1-11 (2011)

11. Qian, W-M: Schur convexity for the ratios of the Hamy and generalized Hamy symmetric functions. J. Inequal. Appl. 2011, Article ID 131 (2011). doi:10.1186/1029-242X-2011-131

12. Chu, Y-M, Xia, W-F, Zhang, X-H: The Schur concavity, Schur multiplicative and harmonic convexities of the second dual form of the Hamy symmetric function with applications. J. Multivar. Anal. 105(1), 412-421 (2012)

13. Rovenţa, I: A note on Schur-concave functions. J. Inequal. Appl. 2012, Article ID 159 (2012) doi:10.1186/1029-242X-2012-159

14. Xia, W-F, Zhan, X-H, Wang, G-D, Chu, Y-M: Some properties for a class of symmetric functions with applications. Indian J. Pure Appl. Math. 43(3), 227-249 (2012)

15. Shi, H-N, Zhang, J: Schur-convexity of dual form of some symmetric functions. J. Inequal. Appl. 2013, Article ID 295 (2013). doi:10.1186/1029-242X-2013-295

16. Wang, BY: Foundations of Majorization Inequalities. Beijing Normal University Press, Beijing (1990) (in Chinese)

17. Shi, H-N: Theory of Majorization and Analytic Inequalities. Harbin Institute of Technology Press, Harbin (2012) (in (hinese)

18. Anderson, GD, Vamanamurthy, MK, Vuorinen, M: Generalized convexity and inequalities. J. Math. Anal. Appl. 335(2), 1294-1308 (2007)

19. Chu, Y-M, Sun, T-C: The Schur harmonic convexity for a class of symmetric functions. Acta Math. Sci. 30B(5), 1501-1506 (2010)

20. Guan, K, Guan, R: Some properties of a generalized Hamy symmetric function and its applications. J. Math. Anal. Appl. 376(2), 494-505 (2011)

10.1186/1029-242X-2013-527

Cite this article as: Shi and Zhang: Some new judgement theorems of Schur geometric and Schur harmonic convexities for a class of symmetric functions. Journal of Inequalities and Applications 2013, 2013:527

\section{Submit your manuscript to a SpringerOpen ${ }^{\circ}$ journal and benefit from:}

- Convenient online submission

- Rigorous peer review

- Immediate publication on acceptance

Open access: articles freely available online

- High visibility within the field

- Retaining the copyright to your article 